WP ECON 12.12

On the welfare loss caused by inequality of opportunity

\author{
Aitor Calo Blanco (U. de A Coruña) \\ J. Ignacio García Pérez (U. Pablo de Olavide)
}

JEL Classification numbers: D31, D63, J71

Keywords: equality of opportunity, income distribution, ex post compensation, welfare loss 


\title{
On the welfare loss caused by inequality of opportunity*
}

\author{
Aitor Calo-Blanco ${ }^{\dagger}$ \\ J.Ignacio García-Pérez \\ University of A Coruña \\ University Pablo de Olavide
}

November, 2012

${ }^{*}$ We would like to thank Antonio Villar, Dirk Van de Gaer, Juan D. Moreno-Ternero, as well as participants to conferences in Moscow, Oslo and A Coruña for their helpful comments. We gratefully acknowledge financial support from the Junta de Andalucía under project SEJ-6882/ECON, and from the Spanish Ministry of Education under project ECO2010-21706. The usual disclaimer applies.

${ }^{\dagger}$ Corresponding author at: University of A Coruña, Dpto. de Analisis Económico y Admon. de Empresas, Campus de Elviña s/n, 15071 A Coruña, Spain. E-mail: aitorcalo@gmail.com 


\begin{abstract}
Based on the ethical principle of equality of opportunity, this paper presents a measure of the welfare loss that is caused by an unfair distribution of a particular outcome (income, health, education, etc). The key idea is that a fair society should produce outcomes that depend on individuals' effort and not on their external circumstances such as gender, socioeconomic background, etc. We propose measuring inequality of opportunity as the welfare loss attributed to the outcome differences among individuals who exert a similar level of effort. Our results are in line with those aspects of fairness literature that give priority to the ex post compensation approach to equality of opportunity. Finally, we present an empirical application for the measurement of the welfare loss in the income distribution in Europe. We have observed a high degree of heterogeneity among European countries. The welfare loss due to inequality of opportunity ranges in those, from basically zero to almost one fifth of their potential welfare.
\end{abstract}

JEL classification: D31, D63, J71.

Keywords: equality of opportunity, income distribution, ex post compensation, welfare loss. 


\section{Introduction}

This paper addresses the measurement of unfairness in the distribution of income, focusing on the unequal treatment of social groups. This kind of analysis involves a mixture of positive and normative economics, taking into account the heterogeneous nature of the elements that influence the explanation of outcome differentials (gender, education, labour status, etc.). Unravelling inequality of opportunity from the rewards differences in agents' characteristics (the yields of diverse human capital levels, say) therefore becomes a critical modelling choice. Our analysis refers to income but is actually applicable to the distribution of any one-dimensional variable that expresses individuals' achievements in a society.

We follow Roemer's well-known approach to equality of opportunity (see Roemer 1993, 1998), which establishes that the distribution of any outcome (income, health, education, etc.) can be regarded as the result of, at least, two different factors: circumstances and effort. Effort has to do with responsibility, and reflects personal decisions such as investment in human capital or lifestyle. Circumstances refer to the individuals' external characteristics for which they cannot be held responsible (such as race, gender, family background, etc.). The key point of this theory is that outcome inequalities that arise from differences in opportunity should be considered socially unfair.

The theory of fairness and responsibility formally defines how social compensation must be implemented in a society, distinguishing between two mainstream principles at the time of administering such a compensation (see Fleurbaey 2008). The so-called principle of compensation states that differences not attributable to responsibility should be eliminated. Conversely, the so-called principle of reward says that inequalities due to responsibility should be left untouched. There is extensive evidence that it 
is impossible to put these two principles into practice simultaneously. ${ }^{1}$

The equality of opportunity perspective presented above is in line with the strand of the literature that focuses on the principle of compensation. However, such a principle can be approached from either an ex ante or an ex post viewpoint (e.g., Fleurbaey 2008 and Ramos and Van de Gaer 2012). From the ex ante perspective there is equality of opportunity if all individuals face the same outcome prospects, no matter their circumstances. The ex post approach, instead, tries to reduce actual outcome differences between individuals who have exerted the same degree of effort. Fleurbaey and Peragine (2012) have shown that the conflict between the compensation and the reward principles is a particular aspect of a deeper tension between ex ante and ex post perspectives. In the present paper we opt to give priority to the ex post compensation approach.

The first part of the present paper presents a theoretically derived methodology that permits to measure the aggregate welfare loss that society experiences due to the existence of inequality of opportunity. We start by describing our reference model, before going on to consider an index of inequality that is decomposable. Next, we show that if one requires a few basic ethical requirements to be satisfied, a closed formula that measures inequality of opportunity can be defined by means of the Theil first index of inequality. Finally, after evaluating the overall level of inequality, we determine the welfare loss that it generates. It must be remembered here that the idea of using an additively decomposable index to allow for a in-depth assessment of segregation is not new. It appears in the works of Theil and Finizza (1971) and Fuchs (1975) among others and, more recently, in Mora and Ruiz-Castillo (2003a, 2003b).

In the second part of the paper we make use of our measure of welfare loss to evaluate the actual cost of inequality of opportunity in all the

\footnotetext{
${ }^{1}$ See Fleurbaey (1994), Bossert (1995), Fleurbaey (2008), and Fleurbaey and Maniquet (2011) for a detailed explanation of the problem.
} 
member states of the European Union prior to the accession of the 10 candidate countries on 1 May 2004 (EU15). More precisely, we will focus on the total annual household income after tax of the 25-66 age group as the reference outcome. External circumstances will be defined by a few personal characteristics that are beyond the individuals' control such as gender, parental background, and labour status. The effort variable will be determined by means of the so-called quantile or tranche approach as defined in Roemer (1993) and Peragine (2004), which is related to individuals' position in their own type income distribution.

Roemer et al. (2003) was one of the first works to provide an empirical assessment of the effect of inequality of opportunity, which included an analysis for the US and 10 European countries of the extent to which income taxation equalises opportunities among young men. This pioneering work was followed by numerous others that measured the level of inequality of opportunity in different countries. Those papers can be classed according to the perspective they adopt. Examples of the ex ante approach are Bourguignon et al. (2007), Ferreira and Gignoux (2011), and Marrero and Rodriguez (2012). The three of them resort to parametric models to estimate the level of inequality of opportunity. Also from the ex ante perspective, Lefranc et al. (2009) and Peragine and Serlenga (2008) analyse various definitions of equality of opportunity in terms of stochastic dominance criteria. On the contrary, the ex post approach is used by Aaberge et al. (2011), Checchi and Peragine (2010) and Pistolesi (2009). The latter uses parametric techniques as well, while the other two works focus on non-parametric models that allow for both, the ex-ante and the ex-post evaluation of equality of opportunity. In the present paper we provide an empirical application of the methodology derived in the theoretical part, and, unlike other references, we show a precise measure of the welfare cost related to inequality of opportunity. Additionally, we empirically evaluate the relevance of both the choice of the types, and the 
effect of income differences that cannot be attributed to circumstances nor responsibility.

We have observed a high degree of heterogeneity in the EU15, with the Nordic countries clearly outperforming the rest of the European nations in terms of both average income and overall inequality, whereas the worst values of the indicators are yield by the Mediterranean Area. Additionally, we have also computed a precise measure of the welfare loss entailed by the existence of inequality of opportunity. We have found that, in the analysis of the 15 countries, there exists a remarkable heterogeneity in terms of welfare cost due to inequality of opportunity, with values ranging from $2 \%$ to one fifth of the potential welfare.

The rest of the paper is organised as follows: Section 2 defines notation and develops the formula to evaluate inequality of opportunity as a social welfare loss; Section 3 presents the data and the empirical results of applying our measure of welfare loss to the European distribution of income; and the final section contains the conclusion.

\section{The model}

\subsection{Preliminaries: inequality, social welfare, and equality of opportunity}

Let us begin with the supposition that we want to assess the welfare content of a given income distribution $\mathbf{x} \in \mathbb{R}_{++}^{n}$ in a homogeneous population that consists of $n$ individuals, by means of a social evaluation function $W: \mathbb{R}_{++}^{n} \rightarrow$ $\mathbb{R}$. Following the works by Atkinson (1970) and Sen (1973) we can define the equally-distributed equivalent income $x^{e}(\mathbf{x})$ as the income level such that $W(\mathbf{x})=W\left(x^{e}(\mathbf{x}) \mathbf{1}^{n}\right)$, where $\mathbf{1}^{n}$ is the $n$-vector all whose components are equal to one. In other words, $x^{e}(\mathbf{x})$ is the income level that, if enjoyed by all members of the society, would yield the same social welfare as the actual distribution. For any inequality-averse social evaluation function we have 
that $x^{e}(\mathbf{x}) \leq \mu(\mathbf{x})$. Therefore, we can define a relative inequality measure as:

$$
I_{W}(\mathbf{x})=1-\frac{x^{e}(\mathbf{x})}{\mu(\mathbf{x})}
$$

where $I_{W}$ depends on the social evaluation function we choose. We can interpret this index, much in the spirit of the Lorenz curve, as a money metric that gives the share of per capita welfare that is lost as a consequence of the unequal distribution. This expression allows for the natural definition of a social evaluation function as follows:

$$
W_{I}(\mathbf{x})=n x^{e}(\mathbf{x})=X[1-I(\mathbf{x})],
$$

where $X=n \mu(\mathbf{x})$ is the aggregate income. In other words, the welfare evaluation of a given distribution discounts from the aggregate income a fraction $X I(\mathbf{x})$ that corresponds to the welfare loss due to inequality. Needless to say, the welfare assessment depends on the inequality index one applies.

Admittedly, choosing a particular index entails special judgments about the distribution of income. According to Eq. (1), one does not need special features, like decomposability properties, to obtain the relative loss due to unfair inequalities. In this sense, Fleurbaey (2008) makes use of the maximin criterion to compute the equally-distributed equivalent income. However, such an approach also comes with important value judgments, since a specific function has to be adopted to define it $\left(x^{e}(\mathbf{x})\right)$. For instance, Fleurbaey (2008) recommends the use of a CES function for empirical analysis. In this case the choice of the constant-elasticity-of-substitution implies a crucial judgment of how society assesses income differences. The set of alternatives would range from the linear case in which there would be no concern for inequality at all, to the maximin criterion in which society would present an infinity aversion to inequality. Being aware of such possibilities, we have 
opted to ground our analysis on inequality indexes that are decomposable rather than on a specific family of social evaluation functions.

Let us suppose that the population can be partitioned into $k$ different subgroups, where for any $j \in\{1, \ldots, k\}$ the number of agents in that subgroup is $n_{j}$, and its income distribution is described by the vector $\mathbf{x}^{j}$. When the inequality measure $I$ is additively decomposable by population subgroups, we can write:

$$
W_{I}(\mathbf{x})=X-X \sum_{j=1}^{k} \omega_{j}(\cdot) I\left(\mathbf{x}^{j}\right)-X I\left[\mu\left(\mathbf{x}^{1}\right) \mathbf{1}^{n_{1}}, \ldots, \mu\left(\mathbf{x}^{k}\right) \mathbf{1}^{n_{k}}\right] .
$$

This expression shows that the welfare evaluation of any distribution $\mathbf{x} \in \mathbb{R}_{++}^{n}$ is measured by the corresponding total income, deflated by two different terms. The first one, $X \sum_{j=1}^{k} \omega_{j}(\cdot) I\left(\mathbf{x}^{j}\right)$, describes the aggregate welfare loss that is due to the inequality within the corresponding population subgroups. Here the terms $\omega_{j}(\cdot)>0$ are the coefficients that determine the relative weight of each population subgroup. The second term of discount, $X I\left(\mu\left(\mathbf{x}^{1}\right) \mathbf{1}^{n_{1}}, \ldots, \mu\left(\mathbf{x}^{k}\right) \mathbf{1}^{n_{k}}\right)$, measures the welfare loss due to the inequality between population subgroups (measured by the dispersion of the mean income of the groups weighted by the corresponding population size).

Let us consider now a society consisting of $N=\{1,2, \ldots, n\}$ individuals who can be classified into $t=1,2, \ldots, \tau$ types, where a type describes the set of agents with the same external circumstances. We can also split the population into $G$ different effort groups or tranches, indexed by $g$. Effort group $g$ consists of those agents in $N$, no matter their types, who have exerted a similar level of effort. A cell $(g, t)$ is the set of agents of type $t$ who belong to effort group $g$. There are $n_{g t}$ agents in cell $(g, t)$ whose income vector is $\mathbf{x}(g, t)$. There are $n_{g}=\sum_{t=1}^{\tau} n_{g t}$ agents in any group $g$, with income $\mathbf{x}(g)=[\mathbf{x}(g, 1), \ldots, \mathbf{x}(g, \tau)]$. Let us consider that $x_{i g}$ describes the income of 
an agent $i \in g$. Therefore, for any $g \in\{1,2, \ldots, G\} ; X_{g}=\sum_{i \in g} x_{i g} \in \mathbb{R}_{++}^{n_{g}}$ is the total income corresponding to those agents in effort group $g$. Finally, an income allocation is a vector $\mathbf{x}=\left(x_{i g}\right)_{i \in N} \in \prod_{g=1}^{G} \mathbb{R}_{++}^{n_{g}}$.

Our aim is to measure the welfare content of an income allocation from the ex post perspective of equality of opportunity. In such a scenario we have that inequality between effort groups is not ethically relevant. Therefore, when the evaluation formula is additively decomposable, Eq. (3) and the equal opportunity principle allows for the definition of the following welfare measure:

$$
V_{I}(\mathbf{x}):=W_{I}(\mathbf{x})+X I\left[\mu\left(\mathbf{x}^{1}\right) \mathbf{1}, \ldots, \mu\left(\mathbf{x}^{G}\right) \mathbf{1}\right]=X\left[1-\sum_{g=1}^{G} \omega_{g}(\cdot) I\left(\mathbf{x}^{g}\right)\right] .
$$

That is, we discard that part of the observed inequality that is due to the differences in agents' level of responsibility.

\section{$2.2 \quad$ A closed evaluation formula}

Eq. (4) allows for a number of alternative specifications, depending on the additively decomposable inequality index we choose. Let us now consider some standard requirements that will lead to a closed evaluation formula.

Our first requirement is that the coefficients that determine the weight of the population subgroups within the decomposition in Eq. (3) add up to one; that is, $\sum_{g=1}^{G} \omega_{g}(\cdot)=1$. When this occurs, we have an exact decomposition of the inequality index and the interpretation of Eq. (3) is much simpler and intuitive, because the within-groups component is just a weighted average of the inequality of the different categories. Moreover, the between-groups component is not independent of those weights when $\sum_{g=1}^{G} \omega_{g}(\cdot) \neq 1$ (Cf. Theil 1967, p. 125).

A regular inequality index can be defined as one that satisfies the following basic properties: symmetry (permuting outcomes does not change 
the value of the index), population replication (replicating a given population does not change the value of the index), Pigou-Dalton's principle of transfers (a mean-preserving progressive transfer reduces inequality), and zero homogeneity (multiplying all components of the distribution by the same positive number has no effect on the value of the index). Shorrocks (1984) showed that any regular and smooth (differentiable) inequality index that is additively decomposable is a member of the generalised entropy family:

$$
G E(\theta)= \begin{cases}\frac{1}{n \theta(\theta-1)} \sum_{i=1}^{n}\left[\left(\frac{x_{i}}{\mu(\mathbf{x})}\right)^{\theta}-1\right], & \text { if } \theta \neq\{0,1\} \\ \frac{1}{n} \sum_{i=1}^{n} \frac{x_{i}}{\mu(\mathbf{x})} \ln \left(\frac{x_{i}}{\mu(\mathbf{x})}\right), & \text { if } \theta=1 \\ \frac{1}{n} \sum_{i=1}^{n} \ln \left(\frac{\mu(\mathbf{x})}{x_{i}}\right), & \text { if } \theta=0 .\end{cases}
$$

Moreover, if we require an exact decomposition of the within-groups term we are left with just two members of the family: the Theil first index, $T$, that corresponds to the value $\theta=1$, and the Theil second index, $T^{*}$, that corresponds to $\theta=0$, which is also known as the mean logarithmic deviation.

So, if we construct a social evaluation function out of a regular inequality index, and we require this index to satisfy additive decomposability plus exact decomposition, our evaluation formula is reduced to just two possibilities: $W_{T}(\mathbf{x})=X[1-T(\mathbf{x})]$ and $W_{T^{*}}(\mathbf{x})=X\left[1-T^{*}(\mathbf{x})\right]$. The main difference between those two indexes is that the role of population and outcome shares is reversed. In the decomposition corresponding to the first index of Theil the coefficients $\omega_{g}(\cdot)$ correspond to outcome shares, whereas in the case of the second index they correspond to population shares.

Finally, let $f_{i g}(\mathbf{x})=\frac{\partial W(\mathbf{x})}{\partial x_{i g}}$ be the marginal social value of agent $i$ in effort group $g$ with income $x_{i g}$. We say that the evaluation function $W$ satisfies the property of minimal equity (e.g., Sen 1973, Villar 2005) when $x_{i g}>x_{h g}$ implies $f_{i g}(\mathbf{x})<f_{h g}(\mathbf{x})$, for each given effort group $g$. That is, it is satisfied when within each effort group we give more weight in social welfare to those 
agents with smaller outcomes. It is easy to see that the Theil first index satisfies this property whereas the second one does not.

We can therefore summarise the above discussion as follows:

Theorem 1 Let $W_{I}: \mathbb{R}_{++}^{n} \rightarrow \mathbb{R}$ be a Social Evaluation Function obtained out of a regular and smooth inequality index. $W_{I}$ satisfies minimal equity and exact additive decomposability if and only if, for all $\mathbf{x} \in \mathbb{R}_{++}^{n}$,

$$
W_{I}(\mathbf{x})=X[1-T(\mathbf{x})],
$$

where $T(\mathbf{x})$ is Theil first index of inequality.

It is easy to see that the associated equality of opportunity welfare measure is given by:

$$
V(\mathbf{x})=\sum_{g=1}^{G} X_{g}[1-T(\mathbf{x}(g))] .
$$

Therefore, we are measuring the welfare content of allocation $\mathbf{x} \in \mathbb{R}_{++}^{n}$ as the aggregate income of all effort groups, each of which is deflated by the level of inequality that it presents, measured by the Theil first index of inequality. Each term $X_{g} T(\mathbf{x}(g))$ gives us the aggregate welfare loss that is due to the unequal distribution of income in group $g$. See Villar (2005) for an alternative characterisation of this formula.

Eq. (6) presents a clear reading in terms of equality of opportunity. Let us remind at this point that the principle of compensation establishes that income differences among individuals with the same level of responsibility should be eliminated. That is to say, social welfare increases whenever an income redistribution within a tranche reduces the value of the Theil index in that specific tranche. Note that our welfare measure establishes that reducing overall inequality within effort groups increases social welfare. Therefore, in a completely 'fair' allocation it must be the case that circumstances are no longer the source of any inequality. This goal clearly follows the line of the ex post compensation approach. 


\subsection{Inequality of opportunity as a social welfare loss}

Let us consider now the measurement of inequality of opportunity that arises from the evaluation formula in Eq. (6). We first determine the welfare loss due to inequality of opportunity (the size of unequal distribution across effort groups), and then we define a simple measure of the relative cost that it entails. To do so we once again apply the decomposability properties of the social evaluation function.

Let each term $X_{g}[1-T[(\mathbf{x}(g)]]$ in Eq. (6) be considered as the welfare measure of income allocation $\mathbf{x}(g)$ for the effort group $g$. Resorting once more to the decomposability property of the index, and taking the constituent groups as the relevant partition, we can state the following:

$$
X_{g}\left[1-T[(\mathbf{x}(g)]]=\sum_{t=1}^{\tau} X_{g t}[1-T(\mathbf{x}(g, t))]-Q_{g}[\mathbf{x}(g)],\right.
$$

where $\mathbf{x}(g, t)$ is the income vector of the $(g, t)$ cell and $X_{g t}$ its aggregate income. For all $t=1,2, \ldots, \tau$; let $T(\mathbf{x}(g, t))$ be the corresponding inequality index and,

$$
Q_{g}(\mathbf{x}(g))=\sum_{t=1}^{\tau} X_{g t} \ln \frac{\mu(g, t)}{\mu(g)}
$$

be the between cells inequality in group $g$. This expression gives us an overall measure of the welfare loss due to inequality of opportunity, attributed to group $g$, as a weighted sum of the relative means (in logs) of the different groups.

Eq. (8) is always positive, and its value increases with the level of inequality within group $g$. If the level of inequality is zero, that means that all individuals have the same income, and hence the value of $Q_{g}(\mathbf{x}(g))$ equals zero. By introducing this expression into Eq. (6) we obtain:

$$
V(\mathbf{x})=\sum_{g=1}^{G} \sum_{t=1}^{\tau} X_{g t}[1-T(\mathbf{x}(g, t))]-\sum_{g=1}^{G} \sum_{t=1}^{\tau} X_{g t} \ln \frac{\mu(g, t)}{\mu(g)}
$$


This equation tells us that the total welfare associated with allocation $\mathbf{x}$ can be understood as the sum of the welfare of all cells that compose the society discounted by the term:

$$
Q(\mathbf{x})=\sum_{g=1}^{G} \sum_{t=1}^{\tau} X_{g t} \ln \frac{\mu(g, t)}{\mu(g)},
$$

which gives us the aggregate welfare loss due to differences in circumstances. This welfare loss consists of a weighted sum of the mean deviations of the cells with respect to their corresponding effort groups, where the weights are given by the corresponding aggregate incomes. Note that $\ln \frac{\mu(g, t)}{\mu(g)}$ is negative when $\mu(g, t)<\mu(g)$, and positive otherwise. Therefore, those cells with mean income above that of their effort group reduce total welfare, whereas those cells with mean income below that of their group increase it.

It is worth stressing that $Q(\mathbf{x})$ is a remarkably simple and intuitive measurement function which does not require much information for computing purposes (in particular it does not require information about the entire distribution).

Function $Q$ is a money metric and, therefore, the welfare assessment will depend on the units in which it is computed. For that reason we opt to define our measure of welfare loss, $L(\cdot)$, as follows:

$$
L(\mathbf{x})=\frac{Q(\mathbf{x})}{V(\mathbf{x})} \times 100 .
$$

That is, $L(\mathbf{x})$ shows us the percentage of the total welfare that is lost due to inequality of opportunity. It is important to stress that Eq. (11) considers that income differences among individuals who belong to the same cell should not be taken into account. This is due to the fact that they cannot be ascribed to inequality of opportunity as defined in our model. Likewise, Checchi and Peragine (2010) apply a smoothing transformation of the income distribution in order to remove such a residual inequality. More precisely, they replace each individual's income by the income mean of the 
cell she belongs to. That is, for all type $t$ and effort group $g$, the smoothed income distribution $\mathbf{x}^{S} \in \mathbb{R}_{++}^{n}$ could be constructed as $x_{i}^{S}=\mu(g, t)$ for all $i \in(g, t)$. Such a transformation would imply that $T\left(\mathbf{x}^{S}(g, t)\right)=0$, and hence the formulae above would become, respectively:

$$
\begin{gathered}
V\left(\mathbf{x}^{S}\right)=X^{S}-Q\left(\mathbf{x}^{S}\right), \\
L\left(\mathbf{x}^{S}\right)=\frac{Q\left(\mathbf{x}^{S}\right)}{X^{S}-Q\left(\mathbf{x}^{S}\right)} \times 100 .
\end{gathered}
$$

Actually, the decomposition presented in this paper would also be valid to compute the welfare loss caused by income differences within cells. Such a loss would be captured by the aggregate welfare loss within cells, which would be measured by the first term in Eq. (9). In that case the new measure of welfare loss would turn to be:

$$
L^{*}(\mathbf{x})=\frac{Q(\mathbf{x})+X_{g t} T(\mathbf{x}(g, t))}{V(\mathbf{x})} \times 100 .
$$

This new expression would give us a measure of the welfare loss which would be taking into account, too, the income inequality that could not be explained either by circumstances or by effort. ${ }^{2}$ Therefore, index $L^{*}(\mathbf{x})$ would always yield a higher value than Eq. (11), and hence we could understand both as the upper and the lower bounds of the real cost of inequality of opportunity.

The present analysis is a reasonable approximation to the measurement of total welfare and the relative loss due to inequality of opportunity. Needless to say, the accuracy of these measures depends on the design of the cells. The larger the number of effort groups, the finer is our definition of the responsibility variable, and the closer is the approximation to its real value. Note that our measure of welfare loss is based on a cardinal measure of

\footnotetext{
${ }^{2}$ For instance, such income differences can be determined by the effect of luck. Luck is a complex factor over which one should distinguish among the specific forms that require full compensation (e.g., Lefranc et al. 2009).
} 
inequality of opportunity. If one wanted to rank income distributions in terms of dominance criteria, she should be aware of the fact that the fewer the number of effort groups, the easier it would be to implement the analysis. ${ }^{3}$

\section{An application: equality of opportunity in European distribution of income}

\subsection{Data}

This section addresses an empirical application of the theoretical framework described above. We aim at studying the income distribution within the former EU15. The data are obtained from the EU-SILC database, ${ }^{4}$ which is a survey that collects timely and comparable cross-sectional and longitudinal multidimensional microdata on income and living conditions. We use data from 2005 since it is the only wave that provides the complete parental module.

In order to carry out our empirical exercise we need to define three elements: (i) individuals' earnings (the outcome); (ii) the set of external circumstances (the types); and (iii) the measurement of responsibility (the effort groups). A detailed explanation of these choices is given below.

Firstly, we have opted to use a per capita measure of the total annual household income after tax as the reference outcome. Such a variable includes all profits (and losses) from labour activities, plus any allowance and earnings derived from property rental. ${ }^{5}$ We have focused on the strictly positive part of the income distribution, since the Theil index cannot be computed over non-positive values. ${ }^{6}$ This variable is not available or is incomplete for four

\footnotetext{
${ }^{3}$ See Peragine (2004) for a deeper discussion of this issue.

${ }^{4}$ European Union Statistics on Income and Living Conditions, a coordinated system of surveys conducted in EU member states.

${ }^{5}$ The variable is constructed as the net version of the disposable equivalent income used by Marrero and Rodriguez (2012). Two income variables could not be deducted from the final value because of the lack of information.

${ }^{6}$ The assumption of limiting the value of the final income is relatively standard (e.g.,
} 
countries, so following Checchi et al. (2010) we have approximated income by means of information on both the gross total earnings, and the existing tax rates in 2004 (see Tables 3, 4, 5 and 6). We have focused on those individuals aged between 25 and 66 .

Secondly, types are determined by characteristics that are assumed to be beyond individuals' control. More precisely, we have used information on gender, parental education (if the individual has at least one parent who has completed upper secondary education), and labour status (if the individual claims to be currently working). Therefore, the total number of types in the baseline scenario adds up to eight. It is important to stress that we have not treated non-employment as a personal choice but rather as part of the agents circumstances. We have favoured such an approach because we understand that non-employment differentials between individuals may derive more from economic and social restrictions than from free choices. ${ }^{7}$ To evaluate the effect of this assumption on the results, we have also computed the welfare loss when such a variable is no longer considered as an external circumstance. Additionally, and in order to check the consistency of our results, we have considered an enlargement of the number of types. We have added two additional circumstances related to health (if the individual reports to be in good health) and experience (if the agent is more than 45 years old).

Finally, when classing agents according to their level of responsibility,

Checchi and Peragine 2010 and Björklund et al. 2012) and implies truncating the lowest tail of the distribution, and hence the final result. The problem is that the elimination of those observations may not have a neutral effect across countries. For instance, there are more negative observations in the Mediterranean countries than in the rest of the sample, so the final value for such an area should be understood as a lower bound of the real welfare cost of inequality of opportunity.

${ }^{7}$ There exists evidence, especially for women, that labour force participation is clearly influenced by social norms and/or the institutional framework. For instance, Algan and Cahuc (2009) argue that the efficient Danish labour market model is unlikely to work in other European countries due to problems of moral hazard. Antecol (2000) and Fernández and Fogli (2009) obtain that cultural factors play an important role in explaining the gender gap in labour force participation. 


\begin{tabular}{|l|c|c|c|}
\hline & Smoothed & Types & Effort Groups \\
\hline \hline $\mathbf{x}_{0}$ & Yes & 8 & 20 \\
\hline $\mathbf{x}^{*}$ & No & 8 & 20 \\
\hline $\mathbf{x}^{* *}$ & Yes & 4 & 20 \\
\hline $\mathbf{x}^{* * *}$ & Yes & 32 & 4 \\
\hline
\end{tabular}

Table 1: Reference income distributions.

we have implemented the Roemer's (1993) assumption that states that individuals in different types have exerted a similar level of effort if they are at the same quantile of their own type income distributions. We have used 20 quantiles to construct the effort groups. This leaves us with a total number of 160 cells in the baseline scenario.

\section{$3.2 \quad$ Results}

We present our results for four different decompositions of the original income distribution $\mathbf{x} \in \mathbb{R}_{++}^{n}$. The first one, $\mathbf{x}_{0} \in \mathbb{R}_{++}^{n}$, is the baseline scenario as described above. Additionally, we have applied a smoothing transformation in which every individual's income has been replaced by the average income of the cell she belongs to. In the second case, $\mathbf{x}^{*} \in \mathbb{R}_{++}^{n}$, we have kept the decomposition but no smooth transformation has been applied. That is to say, income differences within cells have been considered as part of the welfare loss caused by inequality of opportunity. The difference between the baseline scenario and the third counterfactual, $\mathbf{x}^{* *} \in \mathbb{R}_{++}^{n}$, is that in the latter case we have no longer included labour status as a circumstance variable. Finally, in income distribution $\mathbf{x}^{* * *} \in \mathbb{R}_{++}^{n}$, we have increased the number of types up to 32 by considering health and experience as external circumstances as well. In this case, and in order to avoid that some cells were left with no observations, we have reduced the number of effort groups to four. The smooth transformation has also been applied. The characteristics of these reference income distributions are described in Table 1. 


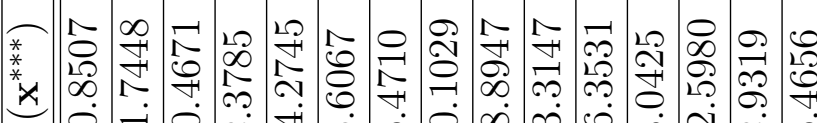

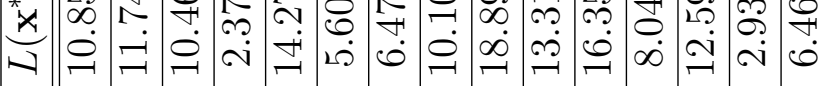

*

凶这织

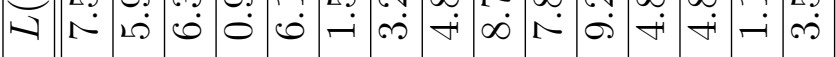

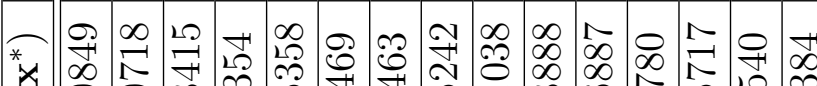

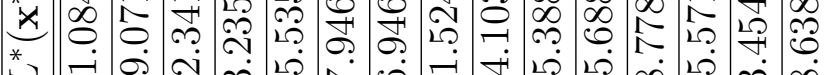

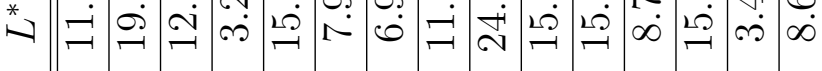

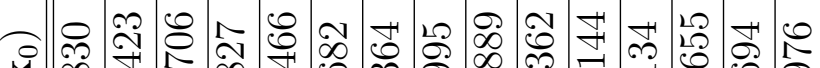

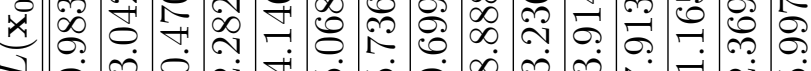

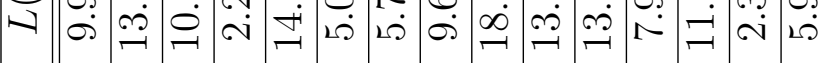

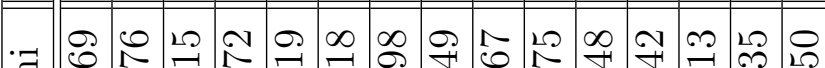

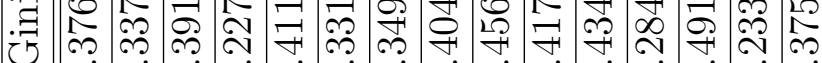

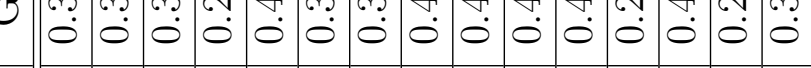

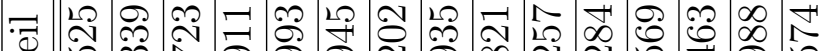

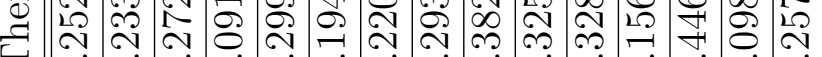

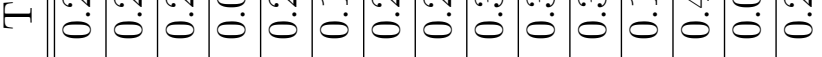

द्वृ

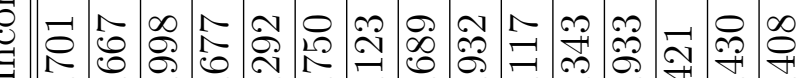

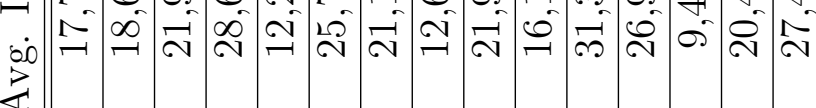

离

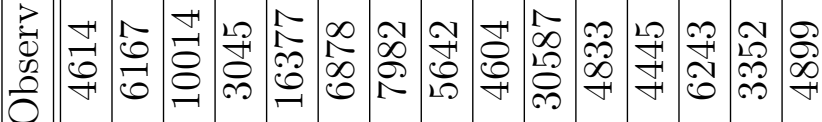

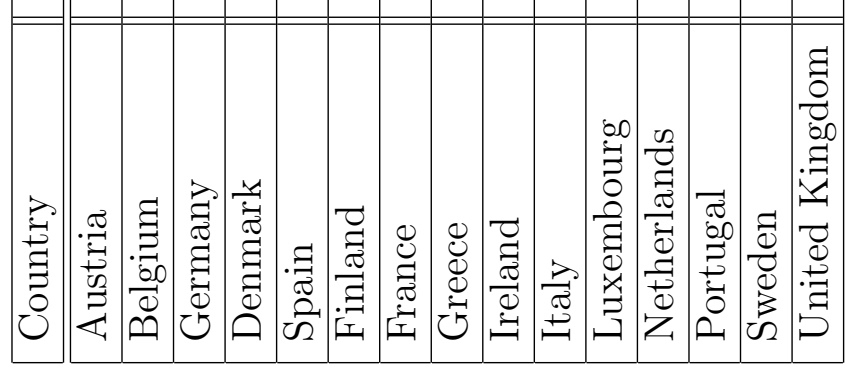

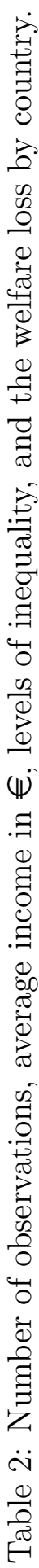


Table 2 summarises the principal statistics of our analysis. It shows, by country, the number of observations, the average income, and the value of both the Gini and the Theil inequality indexes for the baseline scenario. The table also presents the level of welfare loss due to inequality of opportunity for all the income distributions introduced above.

First of all, we observe a high degree of heterogeneity in terms of earnings within the former EU15. For instance, the largest difference between two countries amounts to $€ 20,000$. Likewise, both the Gini coefficient and the Theil first index of inequality yield extremely disparate results across countries. The Nordic region is fairly egalitarian, whereas the countries with the highest levels of inequality within the EU15, with the exception of Luxembourg, are Ireland and the Mediterranean area.

Next, we have computed the explicit welfare loss that is generated by the existence of inequality of opportunity. Figure 1 plots the value of such a loss together with the average household earnings after tax. The most important result that we can draw from the figure is that within the former EU15 there exists a considerable heterogeneity both in income and in welfare loss. Moreover, there appears to be no relationship between the two dimensions whatsoever.

As is usually the case in analyses of this type, Nordic countries present the lowest levels of the welfare loss while at the same time registering relatively high incomes. On the contrary, apart from Belgium and Luxembourg, the worst performance is registered by the usual suspects, namely Italy, Portugal and Spain, with both the lowest average earnings and the highest levels of inequality. Ireland is a special case that presents a high average income but with extremely high welfare loss. Because of the existence of inequality of opportunity such countries are suffering a shortfall of at least one-eighth in their potential welfare. Surprisingly enough, Greece turns out to be an intermediately 'fair' country. ${ }^{8}$ Finally, central European

\footnotetext{
${ }^{8}$ We think that such a relatively good performance may be biased by the specific choice
} 
countries such as France, Germany and Austria yield intermediate results in both dimensions, whereas the United Kingdom and the Netherlands register, alongside Denmark, the highest values of the income dimension.

In Figure 2 we plot, for the baseline scenario, the welfare loss together with the values of the Gini and the Theil indexes. It must be stressed here that, opposite to the measure of the welfare loss, the value of the indexes are also taking into account the level of inequality between effort groups. Although it is clear that a direct relation between overall inequality and the welfare loss must exist, such a relation does not seem to be perfect. For instance, both Spain and Greece present similar levels of overall inequality, albeit the former suffers a higher loss due to inequality of opportunity.

We have also analysed how the results may change with respect to the reference income distribution one chooses. Table 2 also reports the value of the welfare loss computed with the other three decompositions presented in Table 1. First, as we can observe from $L\left(\mathbf{x}^{* * *}\right)$, the results remain virtually equal when we both reduce the number of effort groups, and control for external circumstances related to health and experience. This shows that the variables that drive the final results are gender, parental background, and labour status.

As we have already discussed, we have opted to follow a conservative strategy in which we have assumed that labour status was a full compensation variable. However, it is true that such a consideration is debatable since labour status could be considered as an outcome rather than a circumstance. In order to test the effect of such a variable on the results, $L\left(\mathbf{x}^{* *}\right)$ shows the welfare loss when labour is excluded from the set of circumstances. As we can observe in Figure 1, the welfare cost due to inequality of opportunity decreases in an important proportion (between one third and one half of of the outcome, as many observations with non-positive income have been eliminated from the sample. For instance, a restricted inclusion of all observations with non-positive levels of income means that Greece registers the third highest value of the welfare loss within the EU15. 
its initial value). Therefore, the social judgment on this variable is a crucial choice at the time of evaluating the level of inequality of opportunity. We can interpret these results as the range within which the real cost of inequality of opportunity would lie.

Finally, the results yield by the baseline scenario may also be conditioned by the fact that the income distribution has been transformed in order to suppress all inequalities within cells. This standard assumption has been made because, by definition, such differences should not be attributed to inequality of opportunity. However, if such inequalities stem, for instance, from differences in brute luck, one could also consider them as unfair. ${ }^{9}$ To deal with this discrepancy, we have decided to obtain an upper bound of the welfare cost that includes all those income differences. The results of this final evaluation, $L^{*}\left(\mathbf{x}^{*}\right)$, are also plot in Figure 1. As it is natural, the welfare loss increases when we compute such income differences as inequality of opportunity. However, the new values are just above the original ones. On average, the increase in the welfare loss is slightly above one fourth of the initial value.

\section{Concluding remarks}

There is a well established agreement concerning the fact that a fair society should allow individuals to obtain their final outcomes (income, education, health, etc.) regardless of their external circumstances; in other words, origin should not matter. Such a principle is captured by the notion of equality of opportunity (see Arneson 1989, Cohen 1989, and Roemer 1993, 1998).

In this paper we have studied the effect of inequality of opportunity in terms of welfare loss. First, making use of a decomposable index and some basic ethical requirements, we have derived an evaluation formula that is grounded on the well-known first Theil first index of inequality. Such an

\footnotetext{
${ }^{9}$ More precisely, we refer to lotteries that an individual cannot escape from.
} 
evaluation formula has enabled us to assess the welfare cost generated by the existence of income differences among individuals who have exerted a similar level of effort. Our measure of welfare loss is in line with the part of fairness literature that gives priority to the ex post compensation approach to equality of opportunity.

Next, we have presented an empirical application of our theoretical framework to the analysis of the distribution of per capita household earnings after tax within the former EU15. Our results clearly show that there exists a high degree of heterogeneity among European countries, both in terms of average income and inequality. For instance, Nordic countries perform extremely well in both dimensions, whereas Ireland and the Mediterranean area present a far more worrying situation. We have estimated that the existence of inequality of opportunity may have a negative effect of up to one fifth of the potential welfare.

In closing, we would like to stress two outstanding properties of our theoretical measure of inequality of opportunity. On the one hand, it is an extremely intuitive formula that is grounded on the Theil first index of inequality, which is one of the most widespread methods for measuring inequality. On the other hand, the empirical implementation of the formula is extremely simple, even if we do not have all the information regarding the distribution of the outcome. 


\section{A Appendix}

\section{A.1 Tables and figures}

\begin{tabular}{|c|c|}
\hline Taxable income in $€$ & Tax rate \\
\hline \hline $0-5780$ & 0 \\
\hline $5780-12880$ & 25 \\
\hline $12880-15880$ & 30 \\
\hline $15880-22610$ & 40 \\
\hline $22610-36620$ & 45 \\
\hline $36620-$ & 50 \\
\hline
\end{tabular}

Table 3: Tax rates in the Belgium, 2004.

\begin{tabular}{|c|c|}
\hline Taxable income in $€$ & Tax rate \\
\hline \hline $0-3250$ & 0 \\
\hline $3251-6500$ & 13.3 \\
\hline $6501-9750$ & 19.2 \\
\hline $9751-13000$ & 24.1 \\
\hline $13001-16250$ & 27.6 \\
\hline $16251-19500$ & 28.7 \\
\hline $19501-26000$ & 30.4 \\
\hline $26001-32500$ & 32.7 \\
\hline $32501-39000$ & 34.5 \\
\hline $39001-45500$ & 36.2 \\
\hline $45501-52000$ & 38.9 \\
\hline $52000-65000$ & 42.3 \\
\hline $65001-97500$ & 48.4 \\
\hline $97501-130000$ & 53 \\
\hline $130001-$ & 59.2 \\
\hline
\end{tabular}

Table 4: Tax rates in Denmark, 2004. 


\begin{tabular}{|c|c|}
\hline Taxable income in $€$ & Tax rate \\
\hline \hline $0-12199$ & 0 \\
\hline $12200-17000$ & 9 \\
\hline $17001-20000$ & 14 \\
\hline $20001-32800$ & 19.5 \\
\hline $328001-58200$ & 25 \\
\hline $58201-$ & 32.5 \\
\hline
\end{tabular}

Table 5: Tax rates in Finland, 2004.

\begin{tabular}{|c|c|}
\hline Taxable income in $€$ & Tax rate \\
\hline \hline $0-16265$ & 0 \\
\hline $16266-29543$ & 7.95 \\
\hline $29544-50652$ & 42 \\
\hline $50653-$ & 52 \\
\hline
\end{tabular}

Table 6: Tax rates in the Netherlands, 2004. 


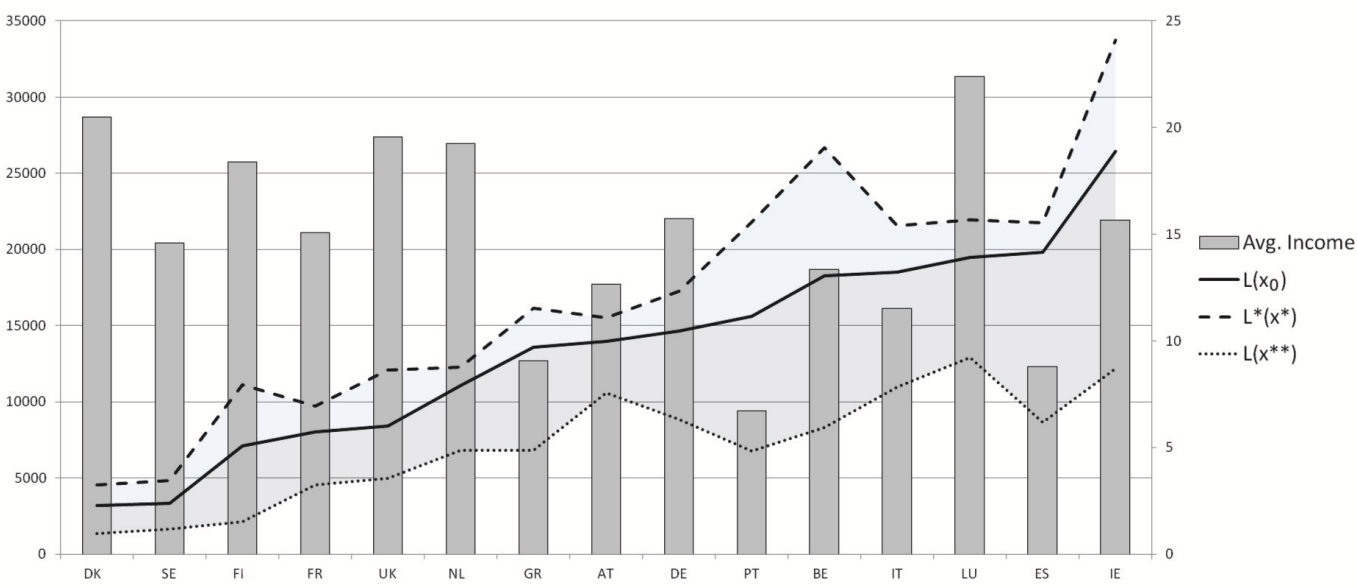

Figure 1: Average income and the welfare loss by country.

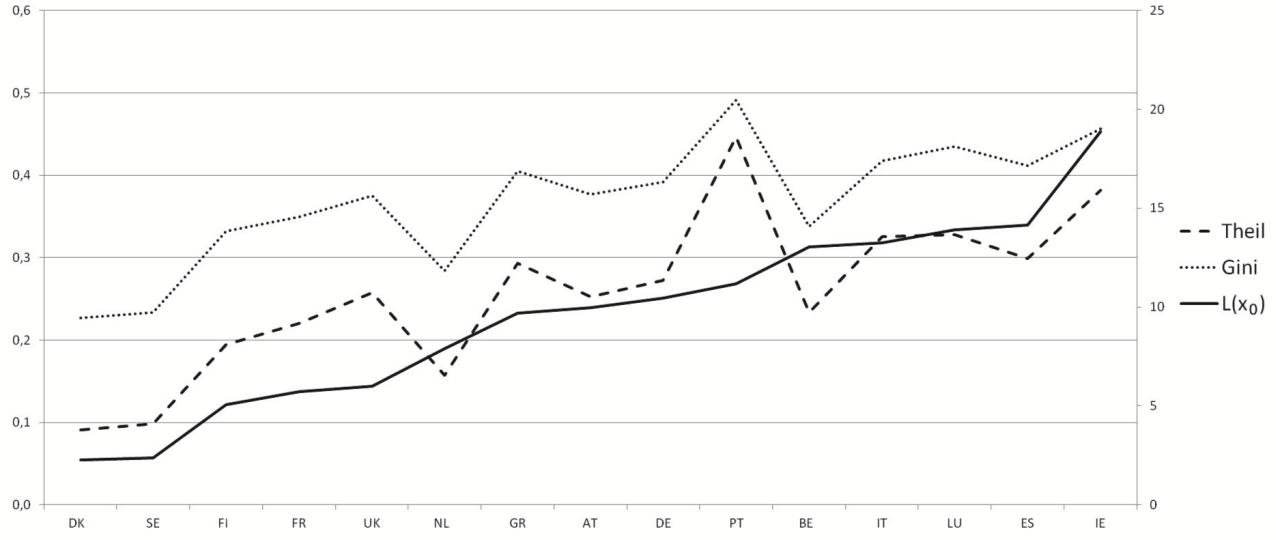

Figure 2: Levels of inequality and the welfare loss by country. 


\section{References}

[1] Aaberge, R., Mogstad, M., Peragine, V.: Measuring long-term inequality of opportunity. Journal of Public Economics 95: 193-204 (2011).

[2] Antecol, H.: An examination of cross-country differences in the gender gap in labor force participation rates. Labour Economics 7: 409-426 (2000).

[3] Algan, Y., Cahuc, P.: Civic virtue and labor market institutions. American Economic Journal: Macroeconomics, 1: 111-45 (2009).

[4] Arneson, R.J.: Equality of opportunity for welfare. Philos Stud 56: 77$93(1989)$.

[5] Atkinson, A.B.: On the measurement of inequality. Journal of Economic Theory 2: 244-263 (1970).

[6] Björklund, A., Jäntti, M., Roemer, J.E.: Equality of opportunity and the distribution of long-run income in Sweden. Social Choice and Welfare 39: 675-696 (2012).

[7] Bossert, W.: Redistribution mechanisms based on individual characteristics. Mathematical Social Sciences 29: 1-17 (1995).

[8] Bourguignon, F., Ferreira, F.H.G., Menéndez, M.: Inequality of opportunity in Brazil. Review of Income and Wealth 53: 585-618 (2007).

[9] Checchi, D., Peragine, V.: Inequality of opportunity in Italy. Journal of Economic Inequality 8: 429-450 (2010).

[10] Checchi, D., Peragine, V., Serlenga, L.: Fair and unfair income inequalities in Europe. Ecineq WP 2010-174 (2010). 
[11] Cohen, G.A.: On the currency of egalitarian justice. Ethics 99: 906-944 (1989).

[12] Fernández, R., Fogli, A.: Culture: An empirical investigation of beliefs, work, and fertility. American Economic Journal: Macroeconomics 1: 146-177 (2009).

[13] Ferreira, F.H.G., Gignoux, J.: The measurement of inequality of opportunity: theory and an application to Latin America. Review of Income and Wealth 57: 622-657 (2011).

[14] Fleurbaey, M.: On fair compensation. Theory and Decision 36: 277-307 (1994).

[15] Fleurbaey, M.: Fairness, responsibility, and welfare. Oxford University Press (2008).

[16] Fleurbaey M., Maniquet, F.: A theory of fairness and social welfare. Cambridge University Press (2011).

[17] Fleurbaey, M., Peragine, V.: Ex ante versus ex post equality of opportunity. Economica 0: 1-13 (2012).

[18] Fuchs, V.: A note on sex segregation in professional occupations. Explorations in Economic Research 31: 105-111 (1975).

[19] Lefranc, A., Pistolesi, N., Trannoy, A.: Equality of opportunity and luck: Definitions and testable conditions, with an application to income in France. Journal of Public Economics 93: 1189-1207 (2009).

[20] Marrero, G., Rodriguez, J.G.: Inequality of opportunity in Europe. Review of Income and Wealth (2012).

[21] Mora, R., Ruiz-Castillo, J.: Additively decomposable segregation indexes. The case of gender segregation by occupations and human 
capital levels in Spain. Journal of Economic Inequality 1: 147-149 (2003a).

[22] Mora, R., Ruiz-Castillo, J.: Gender segregation from birth to occupation. Working Paper 00-63, Economic Series 24, Universidad Carlos III (2003b).

[23] Peragine, V.: Ranking income distributions according to equality of opportunity. Journal of Economic Inequality 2: 11-30 (2004).

[24] Peragine, V., Serlenga, L.: Higher education and equality of opportunity in Italy. Research on Economic inequality 16: 1-31 (2008).

[25] Pistolesi, N.: Inequality of opportunity in the land of opportunities, 1968-2001. Journal of Economic Inequality 7: 411-433 (2009).

[26] Ramos, X., Van de Gaer, D.: Empirical approaches to inequality of opportunity: principles, measures and evidence. CORE Discussion Paper 2012/26 (2012).

[27] Roemer, J.E.: A pragmatic theory of responsibility for the egalitarian planner. Philosophy \& Public Affairs 22: 146-166 (1993).

[28] Roemer, J.E.: Equality of opportunity. Harvard University Press (1998).

[29] Roemer, J.E., Aaberge, R., Colombino, U., Fritzell, J., Jenkins, S.P., Lefranc, A., Marx, I., Page, M., Pommer, E., Ruiz-Castillo, J., San Segundo, M.J., Tranaes, T., Trannoy, A., Wagner, G.G., Zubiri, I.: To what extent do fiscal regimes equalize opportunities for income acquisition among citizens? Journal of Public Economics 87: 539-565 (2003).

[30] Sen, A.: On Economic Inequality. Oxford University Press, Oxford (1973). 
[31] Shorrocks, A.F.: Inequality Decomposition by Population Subgroups. Econometrica 52: 1369-1388 (1984).

[32] Theil, H.: Economics and Information Theory. North-Holland, Amsterdam (1967).

[33] Theil, H., Finizza, A.J.: A note on the measurement of racial integration of schools by means of information concepts. Journal of Mathematical Sociology 1: 187-194 (1971).

[34] Villar, A.: On the Welfare Measurement of Income and Opportunity. Contributions to Theoretical Economics 5: 1 (2005). 